\title{
Plasma Levels of Myocardial MicroRNA-133a Increase by Intraoperative Cytokine Hemoadsorption in the Complex Cardiovascular Operation
}

\author{
Robert Wagner $^{\mathrm{a}, \mathrm{f}, \mathrm{g}}$, Premysl Soucek ${ }^{\mathrm{b}, \mathrm{f}}$, Jiri Ondrasek ${ }^{\mathrm{c}}$, Petr Fila ${ }^{\mathrm{c}}$, Jan Sterba ${ }^{\mathrm{c}}$, \\ Hana Spacilova ${ }^{d}$, Alzbeta Michalcikova ${ }^{\mathrm{e}}$, Tomas Freiberger ${ }^{\mathrm{b}}$, Petr Nemec ${ }^{\mathrm{c}}$
}

\begin{abstract}
Background: Complex cardiovascular procedures may initiate a systemic inflammatory response syndrome (SIRS) with a massive cytokine release, which is involved in postoperative myocardial injury. Intraoperative cytokine hemoadsorption (HA) mitigates the inflammatory response. Micro ribonucleic acids (miRNAs) are emerging as a marker of myocardial injury.
\end{abstract}

Methods: This study evaluated if intraoperative cytokine reduction by HA modulates SIRS and affects myocardial injury as measured by miRNA-126, 223 and miRNA-1, 133a, respectively. Twenty-eight patients were assigned into HA $(\mathrm{n}=15)$ and control $(\mathrm{C})(\mathrm{n}=13)$ groups. HA was performed by integrating CytoSorb ${ }^{\mathrm{TM}}$ into the extracorporeal circuit.

Results: MiRNA-133a plasma levels were increased postoperatively in both groups but were much higher in the HA group than in the $\mathrm{C}$ group at $3 \mathrm{~h}(\mathrm{P}=0.037)$ and $18 \mathrm{~h}(\mathrm{P}=0.017)$ after reperfusion. MiRNA-1 and miRNA-223 plasma levels were significantly increased postoperatively, but did not differ between groups. The vascular miRNA-126 was not affected.

Conclusion: Intraoperative cytokine HA in cardiovascular operations increased the plasma levels of miRNA-133a, suggesting higher myocardial injury.

Manuscript submitted September 6, 2019, accepted October 10, 2019

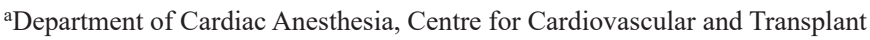
Surgery (CKTCH), Pekarska 53, Brno, Czech Republic

${ }^{b}$ Department of Molecular Genetics, Centre for Cardiovascular and Transplant Surgery $(\mathrm{CKTCH})$, Pekarska 53, Brno, Czech Republic

${ }^{\circ}$ Department of Cardiovascular Surgery, Centre for Cardiovascular and Transplant Surgery (CKTCH), Pekarska 53, Brno, Czech Republic

${ }^{\mathrm{d}}$ Department of Hematological Laboratory, Centre for Cardiovascular and Transplant Surgery (CKTCH), Pekarska 53, Brno, Czech Republic

eDepartment of Psychology, Centre for Cardiovascular and Transplant Surgery (CKTCH), Pekarska 53, Brno, Czech Republic

fThese authors contributed equally to this manuscript.

gCorresponding Author: Robert Wagner, Centre for Cardiovascular and Transplant Surgery (CKTCH), Pekarska 53, 65691 Brno, Czech Republic.

Email: robwag@cktch.cz

doi: https://doi.org/10.14740/jocmr3989
Keywords: MicroRNA; Cytokines; Hemoadsorption; Cardiac operations; Systemic inflammatory response syndrome; Myocardial injury

\section{Introduction}

Cardiac surgery may cause a systemic inflammatory response syndrome (SIRS) with massive cytokine release. Cytokine storm is a major mechanism of adverse defensive reactions after infection or trauma [1]. SIRS after cardiac surgery causes postoperative multiorgan dysfunction, including that of the heart $[2,3]$. A clinical hemoadsorption (HA) device CytoSorb (CytoSorbens Europe, Berlin, Germany) was developed to reduce cytokines when cytokine levels are elevated. A recent meta-analysis concluded that CytoSorb is the most promising current device, based on data from animal studies and initial clinical results $[4,5]$.

Recent studies in adults with different cardiac morbidities have suggested an important role for micro ribonucleic acids (miRNAs) in the pathogenesis of numerous cardiovascular diseases. It has been shown that miRNAs regulate multiple intracellular proteins that function in growth, differentiation, metabolism and apoptosis. MiRNAs are released from cells to mediate intercellular communication after injury [6]. For example, miRNAs have been detected in the plasma under various conditions, including acute coronary syndrome, myocardial infarction, myocardial ischemia-reperfusion injury and after coronary artery bypass grafting (CABG) or heart transplantation, making them a promising biomarker of myocardial injury.

Recent studies have revealed cross-regulation between miRNA and cytokine pathways. It has been shown that miRNA-223 and miRNA-126 dampen inflammatory response, while proinflammatory cytokines such as interleukin (IL)-6, tumor necrosis factor (TNF)- $\alpha$ and others modulate miRNA expression and secretion in response to injury [7-10]. Increased miRNA-1 and/or decreased miRNA-133 levels are associated with myocyte apoptosis in response to ischemia-reperfusion injury [11-14].

Cross-regulation between cytokines and miRNAs during complex cardiac procedures, such as cardiac operations, has not been studied yet. We hypothesized that intraopera- 


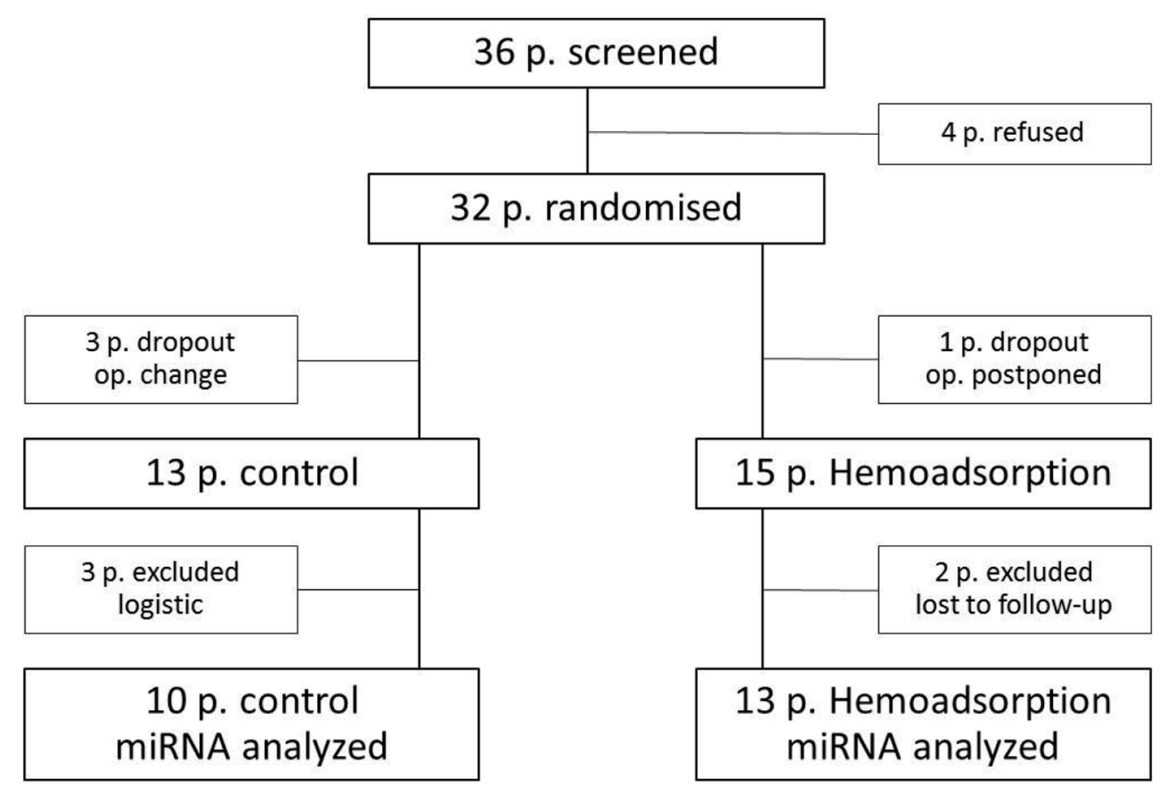

Figure 1. Flow diagram.

tive cytokine reduction by HA modulates SIRS estimated by miRNA-223 and miRNA-126 and affects miRNA-1 and miRNA-133a release after cardioplegic arrest in complex cardiovascular operations.

\section{Materials and Methods}

The study was a randomized, patient-blinded trial performed in a single location with two treatment variables. The study focused on the effects of intraoperative cytokine HA on myocardial, monocyte and vascular miRNAs plasma levels as a primary outcome, and hematological, biochemical and clinical variables as the secondary outcome in 36 patients undergoing cardiac operations. The study was conducted between September 2015 and June 2017. The study was approved by the ethics committee of our institution and was conducted in accordance with the Helsinki Declaration and ICH-GCP guidelines. Per the Consolidated Standards of Reporting Trials guidelines, the trial timeline is shown in Figure 1. Patients excluded from the study included those with rheumatoid arthritis, asthma, cancer, autoimmune disease or those receiving steroidal or nonsteroidal anti-inflammatory drug therapy. Eligible patients were enrolled the day before surgery, and written informed consent was obtained from all patients. Patients were assigned to one of the two treatment arms in a 1:1 ratio using computer-generated system just before surgery. The lab analysts were blinded to patient data and study groups. Laboratory and clinical data were documented in a paper case report form (CRF) daily by the members of the study team. Afterwards, all data were transferred to an electronic data register for research trials in the institutional server database.

Patients were premedicated with pregabalin. Anesthesia was induced with propofol, sufentanil and rocuronium, maintained with the same anesthetics and supplemented with desflurane before and after cardiopulmonary bypass (CPB) to sustain a bispectral index between 37 and 57. Patients were ventilated with an anesthesia machine Drager Zeus (Dragerwerk, Lubeck, Germany) to sustain normocapnia and arterial partial oxygen tension between 13 and $20 \mathrm{kPa}$. All patients were subjected to a routine median sternotomy and standard tepid hypothermic CPB (Stockert S5 perfusion system; Sorin Group, Munich, Germany) with an extracorporeal membrane oxygenator (PRIMO2X; Sorin, Mirandola, Italy). Cold blood cardioplegia was used to preserve the heart. Porcine heparin was administered at $3 \mathrm{mg} / \mathrm{kg}$, and additional doses were administered to maintain a window to prevent clotting for at least $420 \mathrm{~s}$. CPB heparin was neutralized with an equal dose of protamine sulphate. CytoSorb ${ }^{\mathrm{TM}}$ adsorber (CytoSorbens Europe GmbH Berlin, Germany) was installed on the CPB circuit. The device was filled with adsorbent polymer beads composed of porous polymerized divinylbenzene. Beads adsorb hydrophobic molecules in a size range of approximately 10 to $55 \mathrm{kD}$, removing almost all known cytokines. Arterial blood was flowed through cartridge by using side arm coming from arterial line and given back to the venous reservoir. Blood flow through the cartridge was in the range of $300-500 \mathrm{~mL} / \mathrm{min}$, measured by flow sensor (Sorin Group, Munich, Germany). For Ross operation, cryopreserved pulmonary homograft (Tissue bank of the Motol Univ. Hosp. Prague) was implanted to the pulmonary position, and pulmonary autograft was implanted to the aortic position. David operation included the reimplantation of the native aortic valve and the replacement of the diseased aortic root and the ascending aorta.

\section{Blood and tissue sampling}

Five milliliter of blood was obtained from each patient via an 
arterial catheter at five time points: T1: after induction of anesthesia, T2: $3 \mathrm{~h}$ (after skin incision), T3: $6 \mathrm{~h}$ (after intensive care unit (ICU) arrival), T4: $18 \mathrm{~h}$ (the first postoperative day, 5 o'clock a.m.) and T5: $42 \mathrm{~h}$ (the second postoperative day, 5 o'clock a.m.) after myocardial reperfusion (aortic declamping). The blood samples were collected in Sarstedt S-Movette Serum Gel tubes and immediately centrifuged at 1,500 $\mathrm{g}$ for $15 \mathrm{~min}$ at room temperature. The serum was stored at $-70{ }^{\circ} \mathrm{C}$. Myocardial tissue samples were collected from the right auricular appendage or the right atrium before cannulation and after decannulation of the venous cannula. Tissue samples were transferred to siliconized microcentrifuge tubes and immediately frozen in liquid nitrogen. These samples were stored in liquid nitrogen and then at $-70{ }^{\circ} \mathrm{C}$ before processing. Blood was collected for measuring $\mathrm{C}$-reactive protein (CRP), procalcitonin, fibrinogen, thrombocytes, free hemoglobin (fHb) and leukocytes at the same time intervals. A Sysmex XN1000 analyzer (Sysmex Corp., Kobe, Japan) was used for hematological analysis including the SIRS marker NE-SFL (Neutrophil-fluorescent light intensity of the neutrophil area on the white blood cell differential) [15]. FHb was analyzed by spectrophotometry (UV/Vis spectrophotometer WPA S1200+)

To assess cognitive function, the Montreal cognitive assessment (MoCA) was administered the day before the operation and immediately prior to hospital discharge. MoCA assessed eight cognitive domains: short term memory, attention, awareness, executive function, concentration, working memory, language and temporal orientation. MoCA was used in cardiac surgery patients with excellent sensitivity (90\%) and specificity $(87 \%)$. A score of $<26$ was considered mild cognitive impairment $[16,17]$.

RNA extraction and quantitative real-time polymerase chain reaction (qRT-PCR) analysis of miRNA levels

Blood plasma and atrial tissue were stored at $-70{ }^{\circ} \mathrm{C}$ and handled to avoid RNA degradation for all the time before RNA extraction. Total RNA was isolated using mirVana miRNA Isolation Kit (Thermo Fisher-US) according to the manufacturer's instruction without enrichment procedure for small RNAs, and cDNA was immediately synthesized using TaqMan ${ }^{\mathrm{TM}}$ Advanced miRNA cDNA Synthesis Kit (Thermo Fisher-US). TaqMan Advanced miRNAAssays and TaqMan Fast Advanced Master Mix were used for qRT-PCR. MiR-1 was amplified with ID 477820 mir, miR-133a with ID 478511 mir, miR-23b with ID 478602 mir, miR-126 with ID 477887 mir, miR-223 with ID 477983 mir, miR-17-5p with ID $47 \overline{8} 447$ mir, miR-484 with ID $47 \overline{8} 308$ mir and miR-24 with ID $477 \overline{9} 92$ mir assay. The qRT-PCR reactions were loaded into 384-well plates using TECAN FreedomEVO 150 in a final volume of $12 \mu \mathrm{L}$ and run in LightCycler480 II (Roche) in triplicate. The miR-17$5 \mathrm{p}, \mathrm{miR}-484$ and miR-24 were used as references to normalize miRNA levels $[18,19]$. Normalized miRNA levels were analyzed using Advanced Relative Quantification and FitPoints method for $\mathrm{Cp}$ measurements (implemented in LightCycler software). MiRNA levels are presented as ratios of normalized miRNA level in relation to miRNA level in plasma/tissue before surgery.

\section{Statistical analysis}

Demographic and clinical data were summarized by mean and standard deviation or mean and range, expressed through minimum and maximum for metric variables or absolute frequencies for categorical variables. Plasma and tissue miRNA levels were presented as the fold-change relative to T1 (preoperative value), defined as 1,0 . We used the relative quantitative method of $2^{-\Delta \Delta \mathrm{Ct}}$ to measure the differences in expression of selected miRNAs by RT-qPCR [20]. Differences between groups were analyzed by using Student's $t$-test for continuous variables and Fisher's exact test for comparison of frequencies between two groups. Numeric data not normally distributed were compared with a Mann-Whitney U-test. Differences within groups were analyzed using a Wilcoxon test. An alpha of 0.05 was set as the level of statistical significance and indicated with * or \#.

\section{Results}

\section{Patient characteristics}

The selection process for patients included into the study is depicted in Figure 1.

The distribution of sex, age, weight, preoperative cardiac function, medication and Euroscore II did not differ between groups and are shown in Table 1. There were young patients, without serious comorbidities, minimal medications and low Euroscore. Patients had a comprehensive operation of aortic root: Ross operation in $93 \%$ and David operation in $7 \%$. There were no operation-related deaths or permanent serious complications.

Plasma level of myocardial microRNA-133a is more increased by cytokine HA during a comprehensive operation of the aortic root

As shown in Table 2 and Figure 2, plasma levels of miRNA133a increased postoperatively in the both groups but increased much more in the HA group at T2: 63.135 times versus 2.24 times in the control group $(\mathrm{P}=0.037)$ and $\mathrm{T} 4: 62.938$ times versus 3.786 times $(\mathrm{P}=0.017)$.

Plasma level of myocardial microRNA-1 is increased during a comprehensive operation of the aortic root

As shown in Table 2 and Figure 2, there was a significant increase in miRNA-1 plasma levels between presurgery (T1) and at $3 \mathrm{~h}$ (T2) with a peak at $6 \mathrm{~h}$ (T3) and after $18 \mathrm{~h}$ post-reperfusion (T4) in both groups. MiRNA-1 levels in tissue samples were slightly increased postoperatively but the increase was 
Table 1. Characteristics of the Two Groups

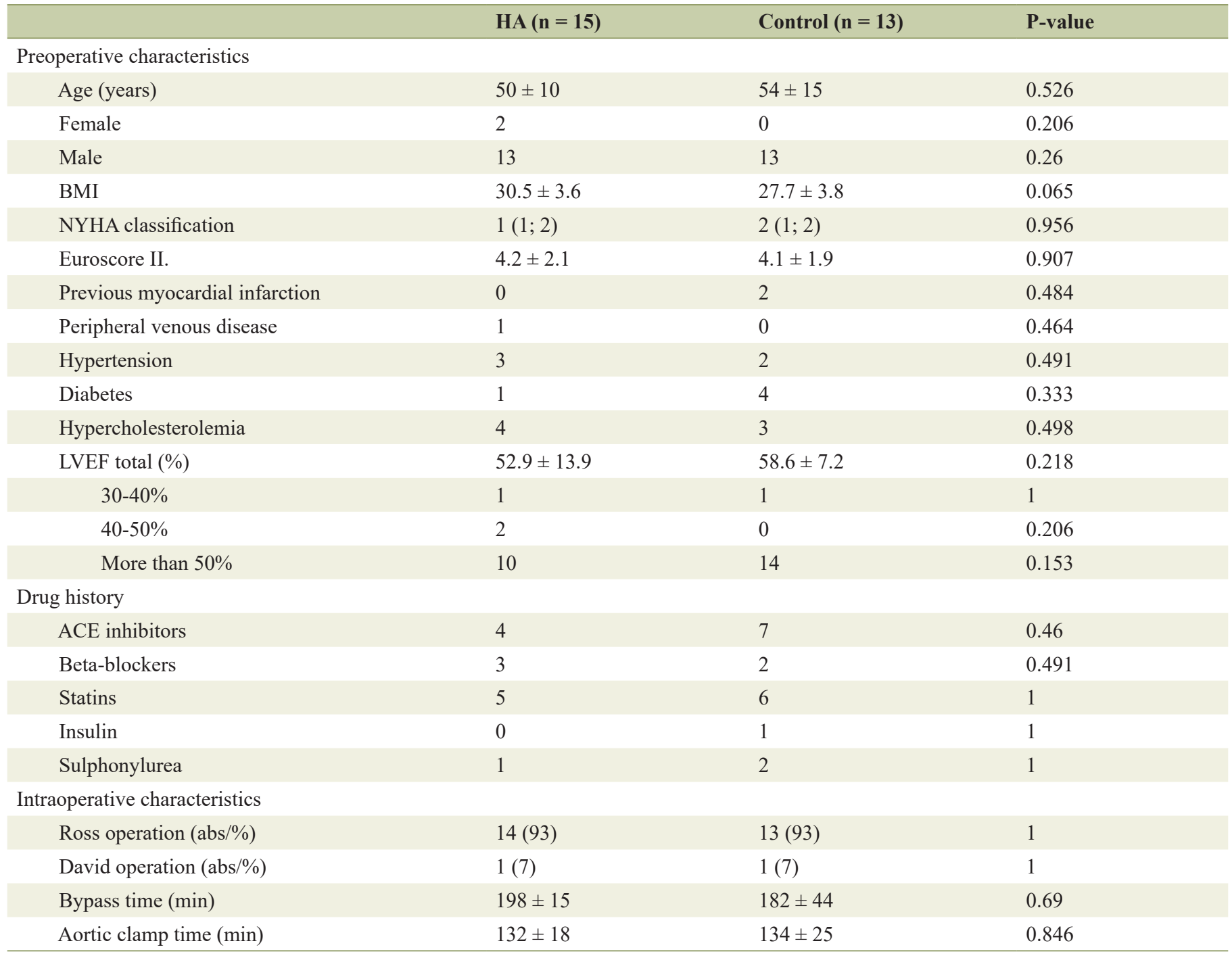

Values are presented as the number (n), percentage (\%) or median (min.; max.). $\mathrm{P}$ values indicating statistical significance were calculated using a $t$-test for continuous variables and a Mann-Whitney U-test for categorical variables. HA: hemoadsorption; BMI: body mass index; NYHA: New York Heart Association classification of heart failure; Euroscore: classification of early (1 month) mortality; LVEF: left ventricle ejection fraction; ACE: angiotensin-converting-enzyme. Bypass time: duration of the extracorporeal circulation; Aortic clamp time: duration of cardioplegic ischemic arrest.

not significantly different between groups.

\section{Plasma level of platelet and monocyte enriched miRNA-223 is increased after comprehensive operations of the aortic root, but vascular miRNA-126 was not affected}

As shown in Table 2, compared to T1, there was a significant increase in miRNA-223 plasma levels in the HA group at T4: 2.143 times $(\mathrm{P}=0.005)$ and at $\mathrm{T} 5: 1.987$ times $(\mathrm{P}=0.01)$. In the $\mathrm{C}$ group, there was a significant decrease at $\mathrm{T} 2: 0.645$ times $(\mathrm{P}=0.036)$, and subsequently followed with increase at T4 and T5 (2.082 times, $\mathrm{P}=0.036$ and 3.16 times, $\mathrm{P}=0.025$, respectively). Plasma levels of vascular miRNA-126 gradually decreased in both groups, but the changes were not sta- tistically significant. There were no significant differences in miRNA-223 and miRNA-126 between the groups.

\section{Secondary outcomes: laboratory and clinical parameters}

We did not observe differences between the groups in inflammatory mediators such as CRP, leukocytes, platelets and fibrinogen levels, and only differences in procalcitonin (PCT) values came close to statistical significance (Table 3). Also, the analysis of white blood cell count and differentials such as neutrophils, immature granulocytes counts and neutrophil SFL (sepsis marker) did not show differences between the groups. FHb increased immediately after the operation: $383 \mathrm{~g} / \mathrm{L}$ (181; $463)$ in the HA group versus $369 \mathrm{~g} / \mathrm{L}(172 ; 477)$ in the control 
Table 2. MiRNA Quantification

\begin{tabular}{|c|c|c|c|c|c|c|c|c|c|c|}
\hline Group & $\mathbf{T}$ & miRNA-23b right atrium & P1 & $\mathbf{P 2}$ & $\begin{array}{l}\text { miRNA-1 } \\
\text { right atrium }\end{array}$ & P1 & $\mathbf{P 2}$ & miRNA-1 blood & P1 & $\mathbf{P 2}$ \\
\hline \multirow[t]{3}{*}{ HA } & 1 & 1 & & & 1 & & & 1 & & \\
\hline & 3 & & & & & & & $16.105(0.723 ; 9,753)$ & 0.451 & $0.004 \#$ \\
\hline & 4 & & & & & & & $9.413(0.116 ; 6,323)$ & 0.369 & $0.013 \#$ \\
\hline \multirow{4}{*}{ Control } & 2 & $0.778(0.144 ; 2.889)$ & & 0.888 & $1.180(0.737 ; 2.683)$ & & 116 & \multicolumn{2}{|l|}{$45.87(1.926 ; 39,471)$} & $0.012 \#$ \\
\hline & 3 & & & & & & & \multicolumn{2}{|l|}{$101.264(1.203 ; 905,424)$} & $0.018 \#$ \\
\hline & 4 & & & & & & & \multicolumn{2}{|l|}{$24.759(0.802 ; 1,041,863)$} & $0.017 \#$ \\
\hline & 5 & & & & & & & \multicolumn{2}{|l|}{$7.412(0.08 ; 1,148)$} & $0.049 \#$ \\
\hline \multirow{4}{*}{ HA } & 2 & $63.135(0.684 ; 790.322)$ & $0.037^{*}$ & 0.004\# & $0.684(0.298 ; 10.491)$ & 0.271 & 0.552 & $0.925(0.446 ; 23.136)$ & 0.077 & 0.65 \\
\hline & 3 & $38.315(1.338 ; 1,024)$ & 0.135 & $0.003 \#$ & $1.25(0.375 ; 47.266)$ & 0.105 & 0.239 & $0.951(0.468 ; 35.055)$ & 0.316 & 0.308 \\
\hline & 4 & $62.938(0.783 ; 1,008)$ & $0.017^{*}$ & $0.004 \#$ & $2.143(0.561 ; 10.337)$ & 0.57 & $0.005 \#$ & \# $0.727(0.269 ; 4.018)$ & 0.443 & 0.875 \\
\hline & 5 & $5.215(0.014 ; 110.887)$ & 0.214 & $0.028 \#$ & $1.987(0.555 ; 9.282)$ & 0.67 & $0.01 \#$ & $0.631(0.006 ; 3.298)$ & 0.877 & 0.182 \\
\hline \multirow[t]{5}{*}{ Control } & 1 & 1 & 1 & 1 & 1 & & & 1 & & \\
\hline & 2 & $2.24(0.408 ; 71.073)$ & & $0.036 \#$ & $0.645(0.194 ; 1.265)$ & & $0.036 \#$ & \# $0.435(0.263 ; 2.911)$ & & 0.214 \\
\hline & 3 & $13.681(1.234 ; 77.345)$ & & $0.018 \#$ & $0.561(0.140 ; 1.725)$ & & 0.398 & $0.62(0.186 ; 2.287)$ & & 0.208 \\
\hline & 4 & $3.786(0.366 ; 62.938)$ & & $0.036 \#$ & $2.082(0.728 ; 4.415)$ & & $0.036 \#$ & \# $0.551(0.169 ; 2.949)$ & & 0.327 \\
\hline & 5 & $0.811(0.174 ; 6.980)$ & & 0.779 & $3.16(0.430 ; 16.599)$ & & $0.025 \#$ & \# $0.619(0.270 ; 6.137)$ & & 0.208 \\
\hline
\end{tabular}

The data are presented as median (min.; max). P1: Comparisons of continuous parameters between groups were computed using a Mann-Whitney U-test. P2: A Wilcoxon paired test was used to assess the significance of time related changes within groups. Plasma and tissue miRNA levels were presented as the fold-change relative to T1 (preoperative value), defined as 1,0 . Statistically significant differences are indicated by an asterisk (*) between groups and a hash tag (\#) within groups. HA: hemoadsorption group. T1: after induction of anesthesia; T2: 3 h; T3: 6 h; T4: 18 h; T5: $42 \mathrm{~h}$ after myocardial reperfusion.

group and then gradually decreased, but there were no differences between the groups $(\mathrm{P}=0.599)$. MoCA showed a onepoint decline, as a difference between preoperative and at the hospital discharge questionnaires: HA: $1(-2 ; 5)$ versus control: $1(-3 ; 5)(\mathrm{P}=0.817)$. We did not find differences in the need for vasoconstrictors and inotropics. Extubation time was approximately $4 \mathrm{~h}(2 ; 10)$ to $5 \mathrm{~h}(3 ; 8)(\mathrm{P}=0.176)$, discharge from the ICU was approximately 2 days $(1 ; 3)$ to 3 days $(1 ; 4)(\mathrm{P}=$ $0.085)$ and discharge from the hospital was approximately 9 days $(7 ; 14)$ to 8 days $(7 ; 15)(\mathrm{P}=0.419)$.

\section{Discussion}

Cardiac surgery is associated with an unpredictable immune system activation demonstrated by an increase in proinflammatory cytokines and decrease in anti-inflammatory cytokines. This imbalance in defensive reaction is historically known as SIRS. SIRS is linked with temporary depression and injury in vital organs such as the heart, lungs, brain and splanchnic or- gans. Despite debates about the clinical importance of SIRS, increasing evidence suggests that after cardiac surgery, SIRS contributes to longer hospitalization in the ICU and higher early postoperative mortality due to organ dysfunction [21]. The knowledge of SIRS pathophysiology has stimulated the development of preventive and therapeutic strategies: prevention includes refinement of surgical techniques and improved biocompatibility of extracorporeal systems or systemic preconditioning [22]. Such methods have resulted in reasonable success, but they appeared to be not fully effective in complex and long lasting surgical procedures. Potential therapy could influence the inflammatory response pharmacologically (corticoids) or decrease activated host defense factors levels and activity either nonspecifically (plasma-cyto-apheresis) or selectively (HA) [23, 24].

Recent studies have indicated a close relationship between cytokines and miRNAs, as miRNA expression is modulated in response to cytokine stimulation [25] and cytokine production is regulated by miRNAs [26]. Because miRNAs play a pivotal role in modulating cardiac function and repair, it is expected 

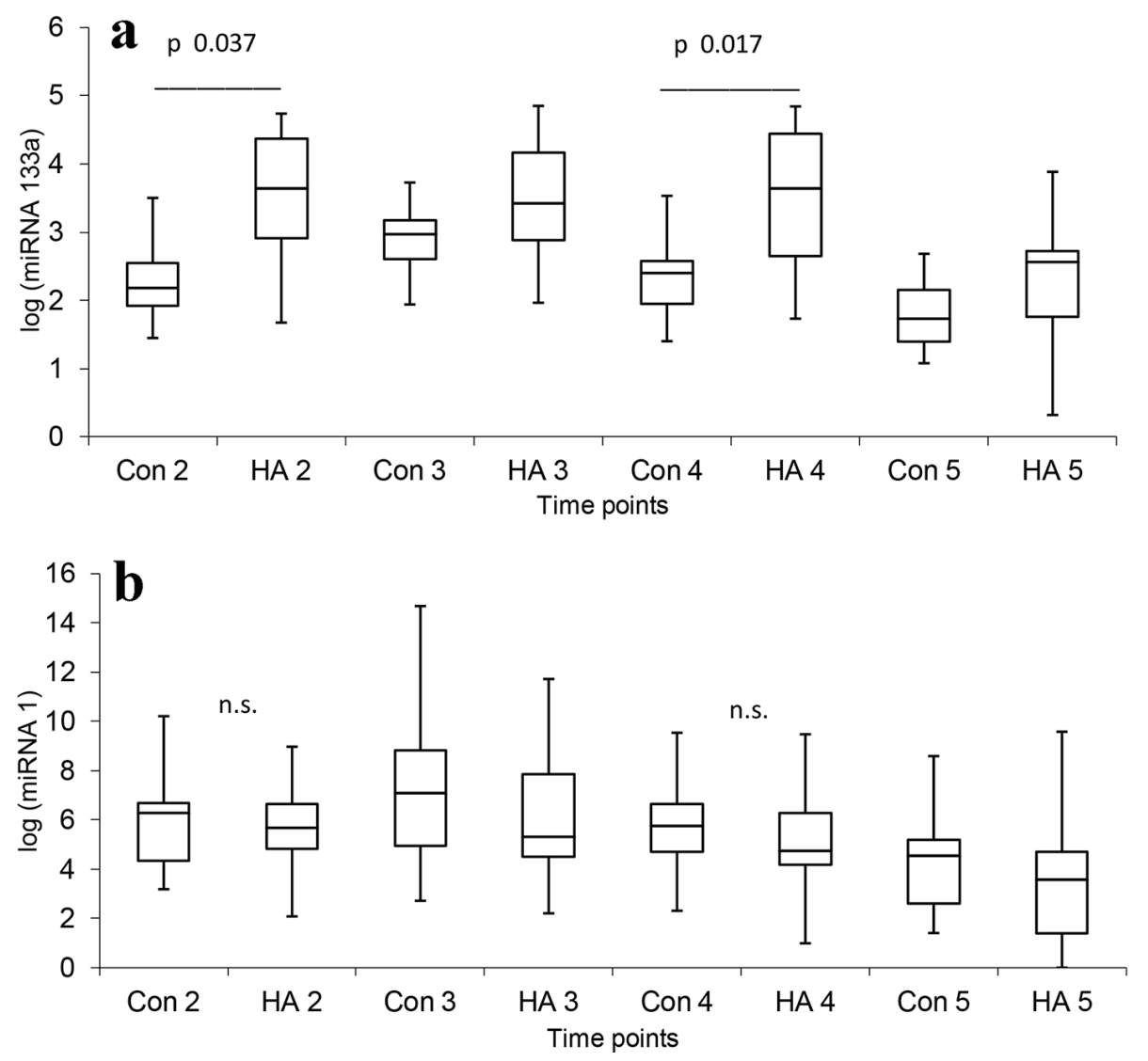

Figure 2. Changes of myogenic miRNAs after surgery. Con: control group; HA: hemoadsorption group. Time points: T1: after induction of anesthesia, T2: $3 \mathrm{~h}$ (after skin incision), T3: $6 \mathrm{~h}$ (after ICU arrival), T4: $18 \mathrm{~h}$ (the first postoperative day, 5 o'clock a.m.) and T5: 42 (the second postoperative day, 5 o'clock a.m.) after aortic declamping. Label a: The plasma levels of miRNA133a were increased postoperatively in both groups but increased much more in the HA group at T2 (Con 2 versus HA 2) and T4 (Con 4 versus HA 4). Label b: The plasma levels of miRNA-1 were increased postoperatively in both groups, but without the difference between groups.

that cytokines modulate myocardial miRNA expression during the postoperative inflammatory response. It has been found that inflammatory cytokines including TNF- $\alpha$, interferon- $\alpha$ (IFN- $\alpha$ ) and IL-1 $\beta$ decrease expression of myogenic miRNAs in muscle inflammation [27].

Several recent studies have found that plasma levels of myogenic miRNAs were elevated after open-heart surgeries, correlated with serum levels of cardiac troponin (cTnI), and responded earlier to myocardial ischemia and infarction than cTnI [28-31]. Through passive release or active secretion, circulating miRNA levels could serve as signaling molecules and have a systemic influence [6]. We also detected high miRNA-1 and miRNA-133a plasma levels in both groups, but miRNA-133a plasma levels were more strongly elevated in the HA group, compared with the control group. The first peak (in hours) of miRNA-133a may come from injured myocytes and the second peak (in days) may be the result of increased expression to initiate myocardial regeneration [32]. If inflammatory cytokines decrease expression of myogenic miRNAs, reduction of cytokines maintained intracellular levels of myogenic miRNAs, which are more released to circulation. Higher miRNA-133a plasma levels are indicative of more extensive myocardial inju- ry or they may reflect some other process mimicking extensive myocardial injury in the HA group compared to control group.

The differences between the myogenic miRNAs in plasma levels could also be that although miRNA-1 and miRNA-133a are simultaneously released from the myocytes, their plasma carriers can be affected differently by the cytokine imbalance or by HA itself, resulting in their different relative levels.

In our study, we measured high interindividual differences in miRNAs levels within groups. This result may misrepresent measured differences, which must be verified using larger study group. A greater homogeneity may be achieved by identifying those diagnoses with more exaggerated defense response such as endocarditis. Myogenic miRNAs originate from the myocardium as well as other muscles. We detected miRNA-1 from atrial tissue before and after CPB, but there was no statistical significance between the groups, likely due to short interval between sample harvesting. Despite this, we hypothesize that the heart was the major source of miRNA-1 and miRNA-133a due to injury after $2 \mathrm{~h}$ resulting in ischemic cardioplegic arrest, while skeletal muscle tissue was normally perfused.

We also measured platelet and monocyte-enriched miRNA-223 because plasma miRNA-223 regulates inflammatory 
Table 3. Postoperative Characteristics

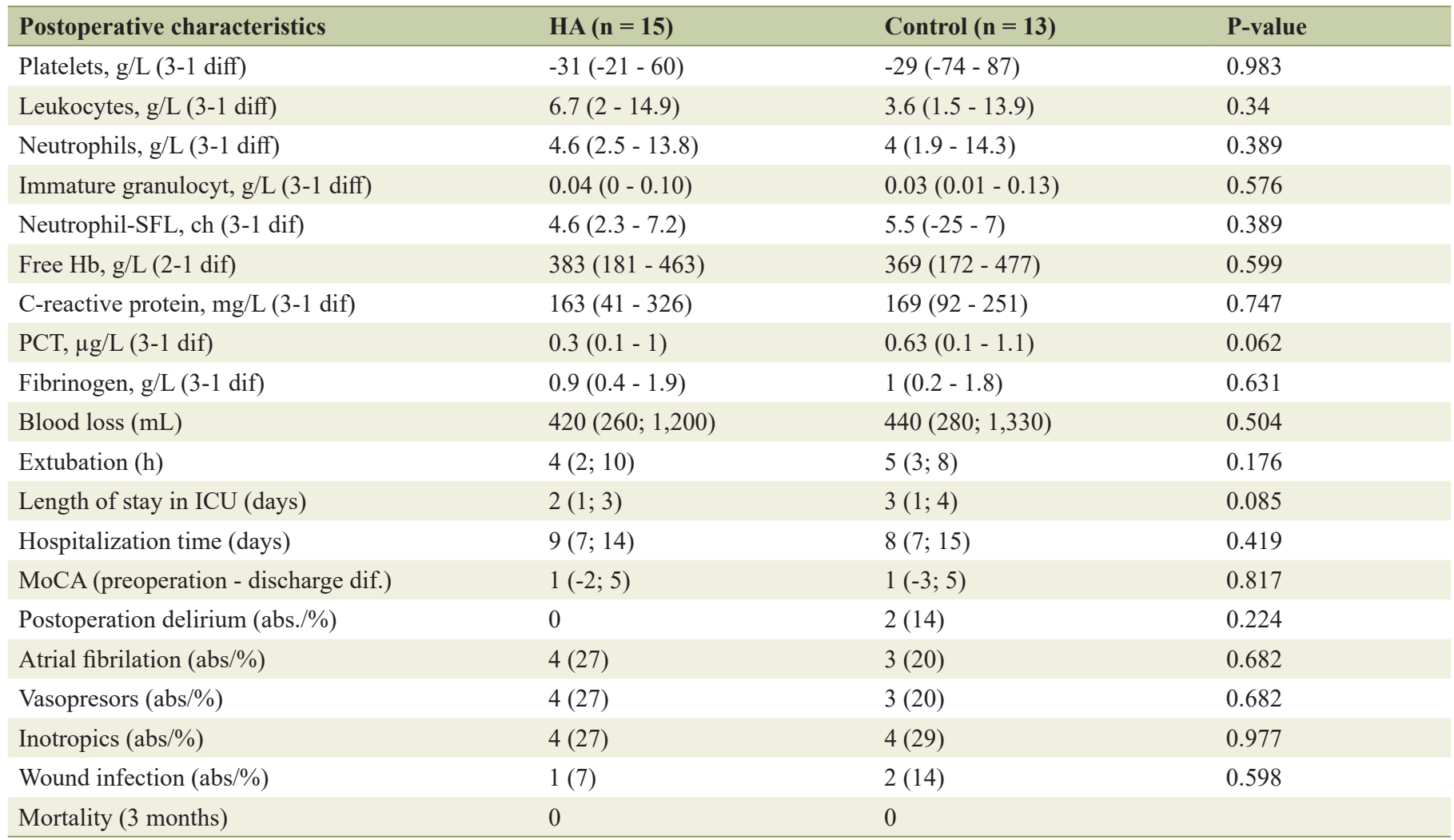

Values are presented as number (abs), percentage (\%), median (min.; max). 3-1 dif: the difference between T3 and T1. Laboratory data are compared between the groups as the difference between the peek value (T3) and the preoperative value (T1). The listed $P$ values of statistical tests were calculated by using Mann-Whitney U-test. HA: hemoadsorption; Neutrophil-SFL (septic marker): the fluorescent light intensity of the neutrophil area on the white blood cell differential scattergram; CRP: C-reactive protein; ICU: intensive care unit; MoCA: Montreal cognitive assessement; PCT: procalcitonin.

responses during cardiac surgery. In fact, miRNA-223 has a homeostatic role in the systemic inflammatory response by dampening inflammatory cytokines via nuclear factor (NF)kappaB downregulation [8]. We detected significant elevation of miRNA-223 the first and second postoperative days for both the groups suggesting that a homeostatic mechanism was activated. We also measured plasma levels of endothelial miRNA-126, which diminish leukocyte trafficking and vascular inflammation by regulating endothelial vascular cell adhesion molecule 1 (VCAM-1) expression [33]. Plasma levels of vascular miRNA-126 gradually decreased in both groups, but the changes were not statistically significant.

There were at least two limitations of this study. First, we did not measure markers of myocardial necrosis or apoptosis. Based on previous studies, we assumed that the HA group had decreased myocardial injury. Second, we do not know whether the observed differences were the result of substances other than cytokines.

\section{Conclusion}

Intraoperative cytokine HA increased the plasma levels of miRNA-133a, suggesting higher myocardial injury. Monocyte
miRNA-223 and vascular miRNA-126 levels were not significantly affected by HA, and postoperative dynamics showed that the homeostatic regulations of the inflammatory response were preserved. Additional research is required to determine whether intraoperative cytokine HA affects myocardial recovery in complex cardiovascular procedures.

\section{Acknowledgments}

We thank Jiri Fabian from the Department of Informatics and Biostatistics, CKTCH for consultation and statistical analyses.

\section{Financial Disclosure}

This work was supported by an institutional grant from the Center for Cardiovascular and Transplant Surgery No. 201607.

\section{Conflict of Interest}

None to declare. 


\section{Informed Consent}

Informed consent was obtained from all individual participants included in the study. This article does not contain any studies with animals performed by any of the authors.

\section{Author Contributions}

WR conceived and designed the study, performed the study, analyzed the data and wrote the paper. SP carried out the PCR measurements, analyzed the data and wrote the paper. OJ performed the surgical procedures and collected the tissue samples. FP performed the surgical procedures and collected the tissue samples. SJ performed the surgical procedures and collected the tissue samples. SH planned the study, carried out the hematological processing and analysis, wrote the paper and analyzed the data. MA performed the psychological testing and analyzed the data. FT planned the study, analyzed the results and wrote the paper. NP performed the surgical procedures, collected the tissue samples and analyzed the results. All authors read, commented on and approved the final manuscript.

\section{Abbreviations}

miRNA: micro ribonucleic acid; HA: hemoadsorption; SIRS: systemic inflammatory response syndrome

\section{References}

1. McGuinness J, Bouchier-Hayes D, Redmond JM. Understanding the inflammatory response to cardiac surgery. Surgeon. 2008;6(3):162-171.

2. Levy JH, Tanaka KA. Inflammatory response to cardiopulmonary bypass. Ann Thorac Surg. 2003;75(2):S715720.

3. Paparella D, Yau TM, Young E. Cardiopulmonary bypass induced inflammation: pathophysiology and treatment. An update. Eur J Cardiothorac Surg. 2002;21(2):232244.

4. Vocelka CR, Jones KM, Mikhova KM, Ebisu RM, Shar A, Kellum JA, Verrier ED, et al. Role of cytokine hemoadsorption in cardiopulmonary bypass-induced ventricular dysfunction in a porcine model. J Extra Corpor Technol. 2013;45(4):220-227.

5. Bernardi MH, Rinoesl H, Dragosits K, Ristl R, Hoffelner F, Opfermann P, Lamm C, et al. Effect of hemoadsorption during cardiopulmonary bypass surgery - a blinded, randomized, controlled pilot study using a novel adsorbent. Crit Care. 2016;20:96.

6. Creemers EE, Tijsen AJ, Pinto YM. Circulating microRNAs: novel biomarkers and extracellular communicators in cardiovascular disease? Circ Res. 2012;110(3):483495.

7. Taganov KD, Boldin MP, Chang KJ, Baltimore D. NF-kappaB-dependent induction of microRNA miR-
146, an inhibitor targeted to signaling proteins of innate immune responses. Proc Natl Acad Sci U S A. 2006;103(33):12481-12486.

8. Poon KS, Palanisamy K, Chang SS, Sun KT, Chen KB, Li PC, Lin TC, et al. Plasma exosomal miR-223 expression regulates inflammatory responses during cardiac surgery with cardiopulmonary bypass. Sci Rep. 2017;7(1):10807.

9. Meyer SU, Thirion C, Polesskaya A, Bauersachs S, Kaiser S, Krause S, Pfaffl MW. TNF-alpha and IGF1 modify the microRNA signature in skeletal muscle cell differentiation. Cell Commun Signal. 2015;13:4.

10. Amado T, Schmolka N, Metwally H, Silva-Santos B, Gomes AQ. Cross-regulation between cytokine and microRNA pathways in $\mathrm{T}$ cells. Eur J Immunol. 2015;45(6):1584-1595.

11. Zhai C, Tang G, Peng L, Hu H, Qian G, Wang S, Yao $\mathrm{J}$, et al. Inhibition of microRNA-1 attenuates hypoxia/reoxygenation-induced apoptosis of cardiomyocytes by directly targeting Bcl-2 but not GADD45Beta. Am J Transl Res. 2015;7(10):1952-1962.

12. Pan Z, Sun X, Ren J, Li X, Gao X, Lu C, Zhang Y, et al. miR-1 exacerbates cardiac ischemia-reperfusion injury in mouse models. PLoS One. 2012;7(11):e50515.

13. Li AY, Yang Q, Yang K. miR-133a mediates the hypoxia-induced apoptosis by inhibiting TAGLN2 expression in cardiac myocytes. Mol Cell Biochem. 2015;400(12):173-181.

14. Izarra A, Moscoso I, Levent E, Canon S, Cerrada I, DiezJuan A, Blanca V, et al. miR-133a enhances the protective capacity of cardiac progenitors cells after myocardial infarction. Stem Cell Reports. 2014;3(6):1029-1042.

15. Briggs C, Longair I, Kumar P, Singh D, Machin SJ. Performance evaluation of the Sysmex haematology XN modular system. J Clin Pathol. 2012;65(11):1024-1030.

16. Nasreddine ZS, Phillips NA, Bedirian V, Charbonneau S, Whitehead V, Collin I, Cummings JL, et al. The Montreal Cognitive Assessment, MoCA: a brief screening tool for mild cognitive impairment. J Am Geriatr Soc. 2005;53(4):695-699.

17. Poole L, Ronaldson A, Kidd T, Leigh E, Jahangiri M, Steptoe A. Pre-operative cognitive functioning and inflammatory and neuroendocrine responses to cardiac surgery. Ann Behav Med. 2016;50(4):545-553.

18. D'Alessandra Y, Devanna P, Limana F, Straino S, Di Carlo A, Brambilla PG, Rubino M, et al. Circulating microRNAs are new and sensitive biomarkers of myocardial infarction. Eur Heart J. 2010;31(22):2765-2773.

19. Marabita F, de Candia P, Torri A, Tegner J, Abrignani S, Rossi RL. Normalization of circulating microRNA expression data obtained by quantitative real-time RT-PCR. Brief Bioinform. 2016;17(2):204-212.

20. Livak KJ, Schmittgen TD. Analysis of relative gene expression data using real-time quantitative PCR and the 2(-Delta Delta C(T)) Method. Methods. 2001;25(4):402408.

21. MacCallum NS, Finney SJ, Gordon SE, Quinlan GJ, Evans TW. Modified criteria for the systemic inflammatory response syndrome improves their utility following cardiac surgery. Chest. 2014;145(6):1197-1203. 
22. Wagner R, Piler P, Bedanova H, Adamek P, Grodecka L, Freiberger T. Myocardial injury is decreased by late remote ischaemic preconditioning and aggravated by tramadol in patients undergoing cardiac surgery: a randomised controlled trial. Interact Cardiovasc Thorac Surg. 2010;11(6):758-762.

23. Wagner R, Piler P, Uchytil B, Halouzka R, Kovaru H, Bobkova M, Nemec P. Systemic inflammatory response syndrome is reduced by preoperative plasma-thromboleukocyte aphaeresis in a pig model of cardiopulmonary bypass. Biomed Pap Med Fac Univ Palacky Olomouc Czech Repub. 2016;160(3):399-406.

24. Kellum JA, Song M, Venkataraman R. Hemoadsorption removes tumor necrosis factor, interleukin-6, and interleukin-10, reduces nuclear factor-kappaB DNA binding, and improves short-term survival in lethal endotoxemia. Crit Care Med. 2004;32(3):801-805.

25. Benes V, Collier P, Kordes C, Stolte J, Rausch T, Muckentaler MU, Haussinger D, et al. Identification of cytokineinduced modulation of microRNA expression and secretion as measured by a novel microRNA specific qPCR assay. Sci Rep. 2015;5:11590.

26. Ando Y, Yang GX, Kenny TP, Kawata K, Zhang W, Huang W, Leung PS, et al. Overexpression of microRNA-21 is associated with elevated pro-inflammatory cytokines in dominant-negative TGF-beta receptor type II mouse. J Autoimmun. 2013;41:111-119.
27. Georgantas RW, Streicher K, Greenberg SA, Greenlees LM, Zhu W, Brohawn PZ, Higgs BW, et al. Inhibition of myogenic microRNAs 1, 133, and 206 by inflammatory cytokines links inflammation and muscle degeneration in adult inflammatory myopathies. Arthritis Rheumatol. 2014;66(4):1022-1033.

28. Zhou X, Mao A, Wang X, Duan X, Yao Y, Zhang C. Urine and serum microRNA-1 as novel biomarkers for myocardial injury in open-heart surgeries with cardiopulmonary bypass. PLoS One. 2013;8(4):e62245.

29. Yao Y, Du J, Cao X, Wang Y, Huang Y, Hu S, Zheng Z. Plasma levels of microRNA-499 provide an early indication of perioperative myocardial infarction in coronary artery bypass graft patients. PLoS One. 2014;9(8):e104618.

30. Yang W, Shao J, Bai X, Zhang G. Expression of Plasma microRNA-1/21/ 208a/499 in myocardial ischemic reperfusion injury. Cardiology. 2015;130(4):237-241.

31. Wang Z, Li X, Shen J, Tian D, Ji Q, Xia L, Lv Q. Plasma microRNAs reflecting cardiac and inflammatory injury in coronary artery bypass grafting surgery. J Surg Res. 2018;224:58-63.

32. Meder B, Katus HA, Rottbauer W. Right into the heart of microRNA-133a. Genes Dev. 2008;22(23):3227-3231.

33. Harris TA, Yamakuchi M, Ferlito M, Mendell JT, Lowenstein CJ. MicroRNA-126 regulates endothelial expression of vascular cell adhesion molecule 1. Proc Natl Acad Sci U S A. 2008;105(5):1516-1521. 